\title{
Optimal Save-Then-Transmit Protocol for Energy Harvesting Wireless Transmitters
}

\author{
Shixin Luo, Rui Zhang, Member, IEEE, and Teng Joon Lim, Senior Member, IEEE
}

\begin{abstract}
In this paper, the design of a wireless communication device relying exclusively on energy harvesting is considered. Due to the inability of rechargeable energy sources to charge and discharge at the same time, a constraint we term the energy half-duplex constraint, two rechargeable energy storage devices (ESDs) are assumed so that at any given time, there is always one ESD being recharged. The energy harvesting rate is assumed to be a random variable that is constant over the time interval of interest. A save-then-transmit (ST) protocol is introduced, in which a fraction of time $\rho$ (dubbed the save-ratio) is devoted exclusively to energy harvesting, with the remaining fraction $1-\rho$ used for data transmission. The ratio of the energy obtainable from an ESD to the energy harvested is termed the energy storage efficiency, $\eta$. We address the practical case of the secondary ESD being a battery with $\eta<1$, and the main ESD being a supercapacitor with $\eta=1$. Important properties of the optimal saveratio that minimizes outage probability are derived, from which useful design guidelines are drawn. In addition, we compare the outage performance of random power supply to that of constant power supply over the Rayleigh fading channel. The diversity order with random power is shown to be the same as that of constant power, but the performance gap can be large. Finally, we extend the proposed ST protocol to wireless networks with multiple transmitters. It is shown that the system-level outage performance is critically dependent on the number of transmitters and the optimal save-ratio for single-channel outage minimization.
\end{abstract}

Index Terms-Energy harvesting, save-then-transmit protocol, outage minimization, fading channel, energy half-duplex constraint, energy storage efficiency, TDMA.

\section{INTRODUCTION}

The operation of communication networks powered either largely or exclusively by renewable sources has become increasingly attractive, both due to the increased desire to reduce energy consumption in human activities at large, and due to necessity brought about by the concept of networking heterogeneous devices ranging from medical sensors on/in the human body to environment sensors in the wilderness [2], [1]. Sensor nodes are powered by batteries that often cannot be replaced because of the inaccessibility of the devices. Therefore, once the battery of a sensor node is exhausted, the node dies. Thus the potentially maintenance-free and virtually perpetual operation offered by energy harvesting, whereby energy is extracted from the environment, is appealing.

S. Luo and T. J. Lim are with the Department of Electrical and Computer Engineering, National University of Singapore (e-mail:\{shixin.luo, eleltj\}@nus.edu.sg).

R. Zhang is with the Department of Electrical and Computer Engineering, National University of Singapore (e-mail: elezhang@nus.edu.sg). He is also with the Institute for Infocomm Research, A*STAR, Singapore.
The availability of an inexhaustible but unreliable energy source changes a system designer's options considerably, compared to the conventional cases of an inexhaustible reliable energy source (powered by the grid), and an exhaustible reliable energy source (powered by batteries). There has been recent research on understanding data packet scheduling with an energy harvesting transmitter that has a rechargeable battery, most of which employed a deterministic energy harvesting model. In [3], the transmission time for a given amount of data was minimized through power control based on known energy arrivals over all time. Structural properties of the optimum solution were then used to establish a fast search algorithm. This work has been extended to battery limited cases in [4], battery imperfections in [5], [6], and the Gaussian relay channel in [7]. Energy harvesting with channel fading has been investigated in [8] and [9], wherein a water-filling energy allocation solution where the so-called water levels follow a staircase function was proved to be optimal.

In scenarios where multiple energy harvesting wireless devices interact with each other, the design needs to adopt a system-level approach [12], [13], [14]. In [13], the medium access control (MAC) protocols for single-hop wireless sensor networks, operated by energy harvesting capable devices, were designed and analyzed. In [14], $N$ energy harvesting nodes with independent data and energy queues were considered, and the queue stability was analyzed under different MAC protocols. An information theoretic analysis of energy harvesting communication systems has been provided in [15], [16]. In [15], the authors proved that the capacity of the AWGN channel with stochastic energy arrivals is equal to the capacity with an average power constraint equal to the average recharge rate. This work has been extended in [16] to the fading Gaussian channels with perfect/no channel state information at the transmitter.

Due to the theoretical intractability of online power scheduling under the energy causality constraint (the cumulative energy consumed is not allowed to exceed the cumulative energy harvested at every point in time), most current research is focused on an offline strategy with deterministic channel and energy state information, which is not practical and can only provide an upper bound on system performance. An earlier line of research considers the problem of energy management, with only causal energy state information, in communications satellites [10], which formulated the problem of maximizing a reward that is linear in the energy as a dynamic programming problem. In [11], energy management policies which stabilize the data queue have been proposed for single-user communication under linear energy-rate approximations. 


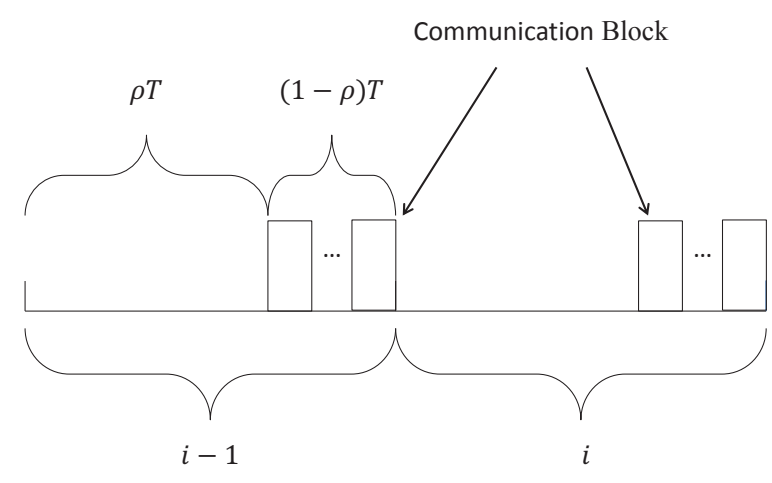

Fig. 1. Save-Then-Transmit (ST) Protocol

In this paper, we focus our study on the design of practical circuit model and transmission protocol for energy harvesting wireless transmitters. To be more specific, we consider a wireless system with one transmitter and one receiver, with the transmitter using a save-then-transmit (ST) protocol (see Fig. 11 to deliver $Q$ bits within $T$ seconds, the duration of a transmission frame. Because rechargeable energy storage devices (ESDs) cannot both charge and discharge simultaneously (the energy half-duplex constraint), an energy harvesting transmitter needs two ESDs, which we call the main ESD (MESD) and secondary ESD (SESD). The transmitter draws power from the MESD for data transmission, over which time the SESD is connected to the energy source and charges up. At the end of transmission for a frame, the SESD transfers its stored energy to the MESD. A fraction $\rho$ (called the saveratio) of every frame interval is used exclusively for energy harvesting by the MESD 1 The energy storage efficiency, denoted by $\eta$, of each ESD may not be 100 percent, and a fixed amount of power $P_{c}$ is assumed to be consumed by the transmitter hardware whenever it is powered up. The frame interval $T$ is assumed to be small relative to the time constant of changes in the ESD charging rate (or energy arrival rate). The energy arrival rate is therefore modeled as a random variable $X$ in Joules/second, which is assumed to be constant over a frame.

Under the above realistic conditions, we minimize the outage probability (to be defined in the next section) over $\rho$, when transmitting over a block fading channel with an arbitrary fading distribution. In this work, we particularize to the case where the MESD is a high-efficiency super-capacitor with $\eta=1$, and the SESD is a low-efficiency rechargeable battery with $0 \leq \eta \leq 1$. Based on the outage analysis, we compare the performance between two system setups: the (new) case with random power supply versus the (conventional) case with constant power supply, over the Rayleigh fading channel. It is shown that energy harvesting, which results in timevarying power availability in addition to the randomness of the fading channel, may severely degrade the outage performance. To be concrete, we further consider exponentially distributed random power, and show that although the diversity order with

\footnotetext{
${ }^{1}$ Note that the energy source can be connected only to either the SESD or the MESD, but not both.
}

exponential power is the same as that with constant power over the Rayleigh fading channel, the outage probability curve may only display the slope predicted by this diversity analysis at substantially higher SNRs.

Finally, we extend the ST protocol for the single-channel case to the general case of wireless network with multiple transmitters. We propose a time division multiple access (TDMA) based ST (TDMA-ST) protocol to allocate orthogonal time slots to multiple transmitters that periodically report to a fusion center. Specifically, we consider two types of source data at transmitters as follows:

- Independent Data: transmitters send independent data packets to the fusion center for independent decoding;

- Common Data: transmitters send identical data packets to the fusion center, where diversity combining is applied to decode the common data.

It is shown that for both cases if the number of transmitters $N$ is smaller than the reciprocal of the optimal transmit-ratio (1$\rho$ ) for the single-channel outage minimization, all transmitters can operate at their individual minimum outage probability. However, as $N$ goes up and exceeds this threshold, the systemlevel outage performance behaves quite differently for the two types of source data.

The rest of this paper is organized as follows. Section $\amalg$ presents the system model. Section [III considers finding the optimal save-ratio for outage minimization and analyzes its various properties. Section IV compares the outage performance between fixed power and random power. Section $\mathrm{V}$ introduces the TDMA-ST protocol for the multi-transmitter case. Section VI shows numerical results. Finally, Section VII concludes the paper.

\section{SySTEM MODEL}

\section{A. Definitions and Assumptions}

The block diagram of the system is given in Fig. 2 The energy harvested from the environment 2 is first stored in either the MESD or the SESD at any given time, as indicated by switch $a$, before it is used in data transmission. The MESD powers the transmitter directly and usually has high power density, good recycle ability and high efficiency, e.g. a supercapacitor [17]. Since the MESD cannot charge and discharge simultaneously, a SESD (e.g. rechargeable battery) stores up harvested energy while the transmitter is on, and transfers all its stored energy to the MESD once the transmitter is off. We assume in the rest of this paper that the SESD is a battery with an efficiency $\eta[$ where $\eta \in[0,1]$. This means that a fraction $\eta$ of the energy transferred into the SESD during charging can be subsequently recovered during discharging. The other $1-\eta$ fraction of the energy is thus lost, due to e.g. battery leakage and/or circuit on/off overhead. The reason of choosing a single-throw switch (switch $a$ in Fig. 2) between the energy harvesting device (EHD) and ESDs is that splitting the harvested energy with a portion going to the SESD, when the transmitter does not draw energy from the MESD, is not

\footnotetext{
${ }^{2}$ Wind, solar, geothermal, etc.

${ }^{3}$ In practice, the battery efficiency can vary from $60 \%$ to $99 \%$, depending on different recharging technologies [18].
} 


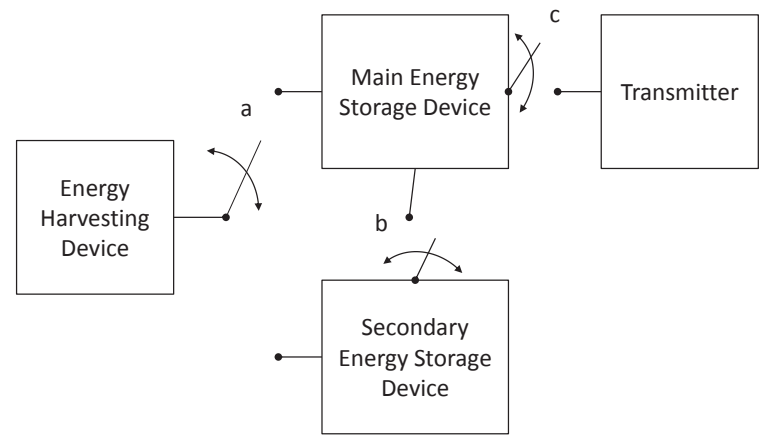

Fig. 2. Energy Harvesting Circuit Model

energy efficient due to the SESD's lower efficiency. Note that at the current stage of research, the optimal detailed structure of an energy harvesting transmitter is not completely known and there exist various models in the literature (see e.g. [8], [6], [9]). The proposed circuit model, given in Fig. 2] provides one possible practical design.

We assume that $Q$ bits of data are generated and must be transmitted within a time slot of duration $T$ seconds (i.e., delay constrained). In the proposed ST protocol, the save-ratio $\rho$ is the reserved fraction of time for energy harvesting by the MESD within one transmission slot. In other words, data delivery only takes place in the last $(1-\rho) T$ seconds of each time slot, which results in an effective rate of $R_{\text {eff }}=\frac{Q}{(1-\rho) T}$ bits/sec. We also allow for a constant power consumption of $P_{c}$ Watts by the transmitter hardware whenever it is powered on. The combined influence of $\rho, \eta$ and $P_{c}$ on outage probability is quantified in this work.

Assume a block-fading frequency-nonselective channel, where the channel is constant over the time slot $T$. Over any time slot, the baseband-equivalent channel output is given by

$$
y=h \cdot x+n,
$$

where $x$ is the transmitted signal, $y$ is the received signal, and $n$ is i.i.d. circularly symmetric complex Gaussian (CSCG) noise with zero mean and variance $\sigma_{n}^{2}$.

For any frame, the ST protocol (cf. Fig. 1) is described as follows:

- During time interval $(0, \rho T]$, harvested energy accumulates in the MESD, which corresponds to the situation that switches $b, c$ are open and $a$ connects to the MESD in Fig. 2,

- From time $\rho T$ to $T$, the transmitter is powered on for transmission with energy from the MESD. Since the transmitter has no knowledge of the channel state, we assume that all the buffered energy in the MESD is used up (best-effort transmission) in each frame. Since the MESD cannot charge and discharge at the same time, the SESD starts to store up harvested energy while the transmitter is on. Referring to Fig. 2 $c$ is closed, $b$ is open and $a$ switches to the SESD;

- At time $T$, the transmitter completes the transmission and powers off. The SESD transfers all its buffered energy to the MESD within a negligible charging time, at efficiency $\eta$. In other words, $b$ is closed and switches $a$ and $c$ are open in Fig. 2

\section{B. Outage Probability}

It is clear that the energy harvesting rate $X$ is a non-negative random variable with finite support, i.e., $0 \leq X \leq P_{H}<$ $\infty$, as the maximum amount of power one can extract from any source is finite. Suppose $f_{X}(x)$ and $F_{X}(x)$ represent its probability density function (PDF) and cumulative distribution function $(\mathrm{CDF})$, respectively. According to the proposed ST protocol, the total buffered energy in the MESD at $t=\rho T$ (the start of data transmission within a transmission slot) is given by

$$
E_{T}=X[\rho+\eta(1-\rho)] T .
$$

Denote $P=\frac{E_{T}}{(1-\rho) T}=X\left[\frac{\rho}{1-\rho}+\eta\right]$ as the average total power, which is constant over the entire transmission period, and $P_{c}$ as the circuit power (i.e. the power consumed by the hardware during data transmission), again assumed constant. The mutual information of the channel (1) conditioned on $X$ and the channel gain $h$ is (assuming $P>P_{c}$ )

$$
R_{T}=\log _{2}\left(1+\frac{\left(P-P_{c}\right)|h|^{2}}{\sigma_{n}^{2}}\right)=\log _{2}\left(1+\left(P-P_{c}\right) \Gamma\right)
$$

where $\Gamma=\frac{|h|^{2}}{\sigma_{n}^{2}}$ with $\operatorname{PDF} f_{\Gamma}(\cdot)$ and $\operatorname{CDF} F_{\Gamma}(\cdot)$.

For a transmitter with energy harvesting capability and working under the ST protocol, the outage event is the union of two mutually exclusive events: Circuit Outage and Channel Outage. Circuit outage occurs when the MESD has insufficient energy stored up at $t=\rho T$ to even power on the hardware for the duration of transmission i.e. $E_{T}<P_{c}(1-\rho) T$ or equivalent $P<P_{c}$. Channel outage is defined as the MESD having sufficient stored energy but the channel realization does not support the required target rate $R_{\text {eff }}=\frac{Q}{(1-\rho) T}$ bits/s.

Recalling that $X \in\left[0, P_{H}\right]$, the probabilities of Circuit Outage and Channel Outage are therefore:

$$
\begin{aligned}
P_{\text {out }}^{\text {circuit }} & =\operatorname{Pr}\left\{P<P_{c}\right\} \\
& =\left\{\begin{array}{cl}
F_{X}[\phi(\cdot)] & \text { if } P_{H}>\phi(\cdot) \\
1 & \text { otherwise. }
\end{array}\right. \\
P_{\text {out }}^{\text {channel }} & =\operatorname{Pr}\left\{\log _{2}\left(1+\left(P-P_{c}\right) \Gamma\right)<R_{\mathrm{eff}}, P>P_{c}\right\} \\
& =\operatorname{Pr}\left\{\Gamma<\frac{2^{R_{\mathrm{eff}}}-1}{P-P_{c}}, P>P_{c}\right\} \\
& =\left\{\begin{array}{cl}
\int_{\phi(\cdot)}^{P_{H}} f_{X}(x) F_{\Gamma}[g(\cdot)] d x & \text { if } P_{H}>\phi(\cdot) \\
0 & \text { otherwise. }
\end{array}\right.
\end{aligned}
$$

where $g\left(\rho, \eta, P_{c}\right)=\frac{\frac{Q}{(1-\rho) T}-1}{x\left[\frac{\rho}{1-\rho}+\eta\right]-P_{c}}$ and $\phi\left(\rho, \eta, P_{c}\right)=\frac{P_{c}}{\frac{\rho}{1-\rho}+\eta}$. Since Circuit Outage and Channel Outage are mutually exclusive, it follows that

$$
\begin{aligned}
P_{\text {out }} & =P_{\text {out }}^{\text {circuit }}+P_{\text {out }}^{\text {channel }} \\
& =\left\{\begin{array}{cl}
F_{X}[\phi(\cdot)]+ \\
\int_{\phi(\cdot)}^{P_{H}} f_{X}(x) F_{\Gamma}[g(\cdot)] d x & \text { if } P_{H}>\phi(\cdot) \\
1 & \text { otherwise. }
\end{array}\right.
\end{aligned}
$$


For convenience, we define

$$
\hat{P}_{\text {out }}\left(\rho, \eta, P_{c}\right)=F_{X}[\phi(\cdot)]+\int_{\phi(\cdot)}^{P_{H}} f_{X}(x) F_{\Gamma}[g(\cdot)] d x
$$

where $\hat{P}_{\text {out }}\left(\rho, \eta, P_{c}\right)<1$ and $P_{H}>\phi(\cdot)$.

Unlike the conventional definition of outage probability in a block fading channel, which is dependent only on the fading distribution and a fixed average transmit power constraint, in an energy harvesting system with block fading and the ST protocol, both transmit power and channel are random, and the resulting outage is thus a function of the save-ratio $\rho$, the battery efficiency $\eta$ and the circuit power $P_{c}$.

\section{Outage Minimization}

In this section, we design the save-ratio $\rho$ for the ST protocol by solving the optimization problem

$$
\text { (P1) : } \min _{0 \leq \rho \leq 1} P_{\text {out }}
$$

i.e. minimize average outage performance $P_{\text {out }}$ in (6) over $\rho$, for any given $\eta \in[0,1]$ and $P_{c} \in[0, \infty)$. Denote the optimal (minimum) outage probability as $P_{\text {out }}^{*}\left(\eta, P_{c}\right)$ and the optimal save-ratio as $\rho^{*}\left(\eta, P_{c}\right)$. Note that $\rho \nearrow 1$ represents transmission of a very short burst at the end of each frame, and the rest of each frame is reserved for MESD energy harvesting. $\rho=0$ is another special case, in which the energy consumed in frame $i$ was collected (by the SESD) entirely in frame $i-1$. (P1) can always be solved through numerical search, but it is challenging to give a closed-form solution for $\rho^{*}\left(\eta, P_{c}\right)$ in terms of $P_{c}$ and $\eta$ in general. We will instead analyze how $\rho^{*}\left(\eta, P_{c}\right)$ varies with $P_{c}$ and $\eta$ and thereby get some insights in the rest of this section.

Proposition 3.1: $P_{\text {out }}\left(\rho, \eta, P_{c}\right)$ in (6) is a non-increasing function of battery efficiency $\eta$ and a non-decreasing function of circuit power $P_{c}$ for $\rho \in[0,1)$. The optimal value of (P1) $P_{\text {out }}^{*}\left(\eta, P_{c}\right)$ is strictly decreasing with $\eta$ and strictly increasing with $P_{c}$.

Proof: Please refer to Appendix A

The intuition of Proposition 3.1 is clear: If $\eta$ grows, the energy available to the transmitter can only grow or remain the same, whatever the values of $\rho$ and $P_{c}$, hence $P_{\text {out }}$ must be non-increasing with $\eta$; if $P_{c}$ grows, the energy available for transmission decreases, leading to higher $P_{\text {out }}$.

\section{A. Ideal System: $\eta=1$ and $P_{c}=0$}

Suppose that circuit power is negligible, i.e. all the energy is spent on transmission, and the SESD has perfect energytransfer efficiency. The condition $P_{H}>P_{c} /\left(\frac{\rho}{1-\rho}+\eta\right)$ is always satisfied, and problem (P1) is simplified to

$$
(\mathrm{P} 2): \min _{0 \leq \rho \leq 1} \int_{0}^{P_{H}} f_{X}(x) F_{\Gamma}\left[\frac{\left(2^{\frac{Q}{(1-\rho) T}}-1\right)(1-\rho)}{x}\right] d x
$$

where the optimal value of $(\mathrm{P} 2)$ is denoted as $P_{\text {out }}^{*}(1,0)$, and the optimal save-ratio is denoted as $\rho^{*}(1,0)$.
Lemma 3.1: The minimum outage probability when $\eta=1$ and $P_{c}=0$ is given by

$$
P_{\text {out }}^{*}(1,0)=\int_{0}^{P_{H}} f_{X}(x) F_{\Gamma}\left[\frac{2^{Q / T}-1}{x}\right] d x
$$

and is achieved with the save-ratio $\rho^{*}(1,0)=0$.

Proof: Please refer to Appendix B

Lemma 3.1 indicates that the optimal strategy for a transmitter that uses no power to operate its circuitry powered by two ESDs with 100 percent efficiency, is to transmit continuously This is not surprising because the SESD collects energy from the environment just as efficiently as the MESD does, and so idling the transmitter while the MESD harvests energy wastes transmission resources (time) while not reaping any gains (energy harvested). However, we will see that this is only true when there is no circuit power and the battery efficiency is perfect.

\section{B. Inefficient Battery: $\eta<1$ and $P_{c}=0$}

When the SESD energy transfer efficiency $\eta<1$ and $P_{c}=$ 0, (P1) becomes

$$
\text { (P3) : } \min _{0 \leq \rho \leq 1} \int_{0}^{P_{H}} f_{X}(x) F_{\Gamma}\left[\frac{\left(2^{\frac{Q}{(1-\rho) T}}-1\right)}{x\left(\frac{\rho}{1-\rho}+\eta\right)}\right] d x
$$

where the optimal value of (P3) is denoted as $P_{\text {out }}^{*}(\eta, 0)$, and the optimal save-ratio is denoted as $\rho^{*}(\eta, 0)$.

Lemma 3.2: When SESD energy transfer efficiency $\eta<1$ and circuit power $P_{c}=0$, the optimal save-ratio $\rho$ has the following properties.

1) A "phase transition" behavior:

$$
\begin{cases}\rho^{*}(\eta, 0)=0, & \eta \in\left[\frac{2^{\frac{Q}{T}}-1}{2^{\frac{Q}{T}}(\ln 2) \frac{Q}{T}}, 1\right) \\ \rho^{*}(\eta, 0)>0, & \eta \in\left[0, \frac{2 \frac{Q}{T}-1}{2^{\frac{Q}{T}}(\ln 2) \frac{Q}{T}}\right)\end{cases}
$$

2) $\rho^{*}(\eta, 0)$ is a non-increasing function of $\eta$, for $0 \leq \eta \leq 1$.

Proof: Please refer to Appendix [C]

According to (9), if the SESD efficiency is above a threshold, the increased energy available to the transmitter if the MESD rather than the SESD collects energy over $[0, \rho T]$ is not sufficient to overcome the extra energy required to transmit at the higher rate $R_{\text {eff }}$ over $(\rho T, T]$. The result is that the optimal $\rho$ is 0 . On the other hand, if $\eta$ is below that threshold, then some amount of time should be spent harvesting energy using the higher-efficiency MESD even at the expense of losing transmission time. Lemma 3.2 quantifies precisely the interplay among $\eta, Q, T$ and $\rho$.

We should note here that even though we consider the case of having two ESD's, by setting $\eta=0$, we effectively remove the SESD and hence our analysis applies also to the single-ESD case. According to (9), if we only have one ESD, the optimal save-ratio is $\rho^{*}(0,0)$, which is always larger than 0 . This is intuitively sensible, because with only one ESD obeying the energy half-duplex constraint, it would be impossible to transmit all the time $(\rho=0)$ because that would leave no time at all for energy harvesting.

\footnotetext{
${ }^{4}$ Except for the time needed in each slot to transfer energy from the SESD to the MESD, which we assume to be negligible.
} 


\section{Non-Zero Circuit Power: $\eta \leq 1, P_{c}>0$}

Non-zero circuit power $P_{c}$ leads to two mutually exclusive effects: (i) inability to power on the transmitter for the $(1-\rho) T$ duration of transmission - this is when $P_{H}<\phi(\cdot)$ in (6); and (ii) higher outage probability if $P_{H}>\phi(\cdot)$ because some power is devoted to running the hardware.

Since $\frac{P_{c}}{\frac{\rho}{1-\rho}+\eta}$ decreases as $\rho$ increases, its maximum value is $\frac{P_{c}}{\eta}$. Therefore, if $P_{H}>\frac{P_{c}}{\eta}$, the transmitter would be able to recover enough energy (with non-zero probability) to power on the transmitter, i.e. $\rho \in[0,1)$. If $P_{H} \leq \frac{P_{c}}{\eta}$, by condition $P_{H} \leq$ $\frac{P_{c}}{\rho}$, save-ratio $\rho$ is required to be larger than $\frac{\frac{P_{c}}{P_{H}}-\eta}{1-\eta+\frac{P_{c}}{P_{H}}}$. In
summary,

- If $P_{c}<P_{H} \eta$

$$
P_{\text {out }}=\hat{P}_{\text {out }}\left(\rho, \eta, P_{c}\right), \quad \forall \rho \in[0,1)
$$

- If $P_{c} \geq P_{H} \eta$

$$
P_{\text {out }}= \begin{cases}1, & \rho \leq \frac{\frac{P_{c}}{P_{H}}-\eta}{1-\eta+\frac{P_{c}}{P_{H}}} \\ \hat{P}_{\text {out }}\left(\rho, \eta, P_{c}\right), & \rho>\frac{\frac{P_{c}}{P_{H}}-\eta}{1-\eta+\frac{P_{c}}{P_{H}}}\end{cases}
$$

If $P_{c} \geq \eta P_{H}$, referring to 10 , we may conclude that $\rho^{*}\left(\eta, P_{c}\right)>\frac{\frac{P_{c}}{P_{H}}-\eta}{1-\eta+\frac{P_{c}}{P_{H}}}$ due to the need to offset circuit power consumption. If $P_{c}{ }^{H}<\eta P_{H}$, theoretically, the transmitter is able to recover enough energy (with non-zero probability for all $\rho \in[0,1))$ to transmit.

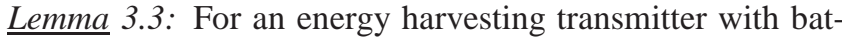
tery efficiency $\eta$ and non-zero circuit power $P_{c}$,

$$
\eta-\frac{P_{c}}{P_{H}}<\frac{2^{\frac{Q}{T}}-1}{2^{\frac{Q}{T}}(\ln 2) \frac{Q}{T}} \Longrightarrow \rho^{*}\left(\eta, P_{c}\right)>0 .
$$

Proof: Please refer to Appendix D

Intuitively, the smaller the circuit power, the more energy we can spend on transmission; the larger the battery efficiency is, the more energy we can recover from energy harvesting. Small circuit power and high battery efficiency suggest continuous transmission $\left(\rho^{*}\left(\eta, P_{c}\right)=0\right)$, which is consistent with our intuition. According to Lemma 3.3. larger circuit power may be compensated by larger ESD efficiency (when the threshold for $\eta$ is smaller than 1). A non-zero save-ratio is only desired if there exists significant circuit power to be offset or substantial ESD inefficiency to be compensated. The threshold depends on required transmission rate.

Remark 3.1: It is worth noticing that if we ignore the battery inefficiency or set $\eta=1$, Lemma 3.3 could be simplified as

$$
P_{c}>\frac{2^{\frac{Q}{T}}(\ln 2) \frac{Q}{T}-2^{\frac{Q}{T}}+1}{2^{\frac{Q}{T}}(\ln 2) \frac{Q}{T}} P_{H} \quad \Longrightarrow \quad \rho^{*}\left(1, P_{c}\right)>0
$$

where only circuit power $P_{c}$ impacts the save-ratio. Since the MESD and the SESD are equivalent $(\eta=1)$, harvesting energy using the MESD is not the reason for delaying transmission. Instead, $\rho^{*}>0$ when $P_{c}$ is so large that we should transmit over a shorter interval at a higher power, so that the actual transmission power minimizes $P_{\text {out }}$. Circuit power similarly determined the fundamental tradeoff between energy efficiency and spectral efficiency (data rate) in [19], in which it was shown that with additional circuit power making use of all available time for transmission is not the best strategy in terms of both energy and spectral efficiency. In this paper, outage is minimized through utilizing available (random) energy efficiently, wherein circuit power causes a similar effect.

\section{DIVERSITY ANALYSIS}

The outage performance of wireless transmission over fading channels at high SNR can be conveniently characterized by the so-called diversity order [20], which is the high-SNR slope of the outage probability determined from a SNR-outage plot in the log-log scale. Mathematically, the diversity order is defined as

$$
d=-\lim _{\bar{\gamma} \rightarrow \infty} \frac{\log _{10}\left(P_{\text {out }}\right)}{\log _{10}(\bar{\gamma})}
$$

where $P_{\text {out }}$ is the outage probability and $\bar{\gamma}$ is the average SNR.

Diversity order under various fading channel conditions has been comprehensively analyzed in the literature (see e.g. [20] and references therein). Generally speaking, if the fading channel power distribution has an accumulated density near zero that can be approximated by a polynomial term, i.e., $\operatorname{Pr}\left(|h|^{2} \leq \epsilon\right) \approx \epsilon^{k}$, where $\epsilon$ is an arbitrary small positive constant, then the constant $k$ indicates the diversity order of the fading channel. For example, in the case of Rayleigh fading channel with $\operatorname{Pr}\left(|h|^{2} \leq \epsilon\right) \approx \epsilon$, the diversity order is thus 1 according to 13 .

However, the above diversity analysis is only applicable to conventional wireless systems in which the transmitter has a constant power supply. Since energy harvesting results in random power availability in addition to fading channels, the PDF of the receiver SNR due to both random transmit power and random channel power may not necessarily be polynomially smooth at the origin (as we will show later). As a result, the conventional diversity analysis with constant transmit power cannot be directly applied. In this section, we will investigate the effect of random power on diversity analysis, as compared with the conventional constant-power case. For clarity, in the rest of this section, we consider the ideal system with $\eta=1$ and $P_{c}=0$, and the Rayleigh fading channel with $\mathbb{E}[\Gamma]=\mathbb{E}\left[\frac{|h|^{2}}{\sigma_{n}^{2}}\right]=\frac{\sigma_{h}^{2}}{\sigma_{n}^{2}}=\lambda_{\gamma}$.

From (5) and (6), the outage probability when $\eta=1$ and $P_{c}=0$ is given by

$$
P_{\text {out }}=\operatorname{Pr}\left\{\log _{2}(1+P \Gamma)<\frac{Q}{(1-\rho) T}\right\} .
$$

Based on Lemma 3.1 the minimum outage probability is achieved with the save-ratio $\rho=0$. Therefore, the outage probability is simplified as 5

$$
P_{\text {out }}^{*}=\operatorname{Pr}\{P \Gamma<C\}=\int_{0}^{\infty} \int_{0}^{\frac{C}{P}} f_{P}(p) f_{\Gamma}(\gamma) d \gamma d p
$$

${ }^{5}$ For convenience, $P_{\text {out }}^{*}$ is used to represent $P_{\text {out }}^{*}(1,0)$ in the rest of this section. 
where $C=2^{\frac{Q}{T}}-1$ and the last equality comes from the assumption of Rayleigh fading channel so the $\Gamma$ is exponential distributed. It is worth noting that in this case with $\eta=1$ and $\rho=0$, according to (2), the energy arrival rate $X$ and the average total power $P$ are identical and thus can be used interchangeably.

Clearly, the near-zero behavior of $P_{\text {out }}^{*}$ critically depends on the PDF of random power $f_{P}(p)$, while intuitively we should expect that random power can only degrade the outage performance. We choose to use the Gamma distribution to model the random power $P$, because the Gamma distribution models many positive random variables (RVs) [21], [22]. The Gamma distribution is very general, including exponential, Rayleigh, and Chi-Square as special cases; furthermore, the PDF of any positive continuous RV can be properly approximated by the sum of Gamma PDFs. Supposing that $P$ follows a Gamma distribution denoted by $P \sim \mathcal{G}\left(\beta, \lambda_{p}\right)$, then its $\mathrm{PDF}$ is given by

$$
f_{P}(p)=\frac{p^{\beta-1} \exp \left(-\frac{p}{\lambda_{p}}\right)}{\lambda_{p}^{\beta} \Gamma(\beta)} U(p)
$$

where $U(\cdot)$ is the unit step function, $\Gamma(\cdot)$ is the Gamma function, and $\beta>0, \lambda_{p}>0$ are given parameters. Referring to [23, Lemma 2], which gives the distribution of the product of $m$ Gamma RVs, the outage probability in (15) can be computed as

$$
P_{\text {out }}^{*}=\frac{1}{\Gamma(\beta)} G_{13}^{21}\left(\frac{C \lambda_{\gamma}}{\lambda_{p}} \mid \begin{array}{l}
1 \\
1, \beta, 0
\end{array}\right)
$$

where $G(\cdot)$ is the Meijer G-function [21].

Meijer G-function can in general only be numerically evaluated and does not give much insights about how random power affects the outage performance. Next, we further assume that the random power $P$ is exponentially distributed (as a special case of Gamma distribution with $\beta=1$ ) to demonstrate the effect of random power.

Lemma 4.1: Suppose that $P$ is exponentially distributed with mean $\lambda_{p}$, the channel is Rayleigh fading with $\mathbb{E}[\Gamma]=$ $\frac{\sigma_{h}^{2}}{\sigma_{n}^{2}}=\lambda_{\gamma}$, and thus the average received $\operatorname{SNR} \bar{\gamma}=\lambda_{p} \lambda_{\gamma}=$ $\frac{\lambda_{p}^{n} \sigma_{h}^{2}}{\sigma_{n}^{2}}$. The minimum outage probability $P_{\text {out }}^{*}$, under an ideal system with $\eta=1$ and $P_{c}=0$, is given by

$$
P_{\text {out }}^{*}=\sum_{k=0}^{\infty} \frac{C^{k+1}}{(k !)^{2}(k+1) \bar{\gamma}^{k+1}}\left[\frac{1}{k+1}-\ln \frac{C}{\bar{\gamma}}+2 \psi(k+1)\right]
$$

where $\psi(x)$ is the digamma function [24] and $\ln (\cdot)$ represents the natural logarithm.

Proof: Please refer to Appendix E

In the asymptotically high-SNR regime, we can approximate $P_{\text {out }}^{*}$ by taking only the first term of $(18)$ as

$$
P_{\text {out }}^{*} \approx \frac{C}{\bar{\gamma}}\left(1-\ln \frac{C}{\bar{\gamma}}+2 \psi(1)\right) \approx \frac{\ln \bar{\gamma}}{\bar{\gamma}} .
$$

${ }^{6} \mathrm{We}$ assume that high SNR is achieved via decreasing noise power $\sigma_{n}^{2}$, while fixing the average harvested energy.

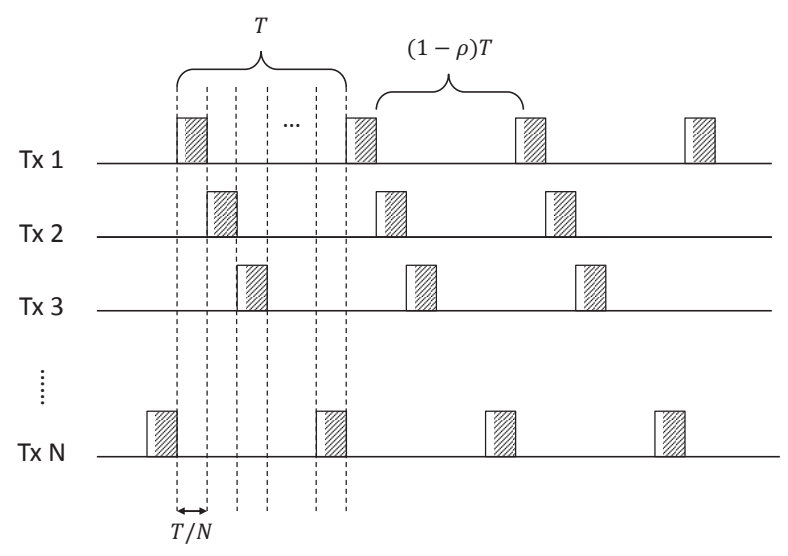

Fig. 3. TDMA based ST (TDMA-ST)

As observed, $P_{\text {out }}^{*}$ decays as $\bar{\gamma}^{-1} \ln (\bar{\gamma})$ rather than $\bar{\gamma}^{-1}$ as in the conventional case with constant power, which indicates that the PDF of the receiver SNR is no longer polynomially smooth near the origin. Hence, the slope of $P_{\text {out }}^{*}$ in the SNR-outage plot, or the diversity order, will converge much more slowly to $\bar{\gamma}^{-1}$ with SNR than in the constant-power case, suggesting that random energy arrival has a significant impact on the diversity performance. More specifically, we obtain the diversity order in the case of exponentially distributed random power as

$$
d=-\lim _{\bar{\gamma} \rightarrow \infty} \frac{-\log _{10} \bar{\gamma}+\log _{10}(\ln \bar{\gamma})}{\log _{10} \bar{\gamma}}=1
$$

which is, in principle, the same as that over the Rayleigh fading channel with constant power. We thus conclude that diversity order may not be as meaningful a metric of evaluating outage performance in the presence of random power, as in the conventional case of constant power.

\section{Multiple Transmitters}

In this section, we extend the ST protocol for the singlechannel case to the more practical case of multiple transmitters in a wireless network, and quantify the system-level outage performance as a function of the number of transmitters in the network.

\section{A. TDMA-ST}

We consider a wireless network with $N$ transmitters, each of which needs to transmit $Q$ bits of data within a time frame of duration $T$ seconds to a common fusion center (FC). It is assumed that each transmitter is powered by the same energy harvesting circuit model as shown in Fig. 2, and transmits over the baseband-equivalent channel model given in (11). We also assume a homogeneous system setup, in which the channel gains, energy harvesting rates or additive noises for all transmitter-FC links are independent and identically distributed (i.i.d).

In order to allow multiple transmitters to communicate with the FC, we propose a TDMA based ST (TDMA-ST) protocol as follows (cf. Fig. 3): 
- Every frame is equally divided into $N$ orthogonal time slots with each slot equal to $\frac{T}{N}$ seconds.

- Assuming perfect time synchronization, each transmitter is assigned a different (periodically repeating) time slot for transmission, i.e., in each frame, transmitter $i$ is allocated the time slot $\left[\frac{(i-1)}{N} T, \frac{i}{N} T\right), 1 \leq i \leq N$.

- Assuming $\rho_{i}=\rho$ for all $i$ 's, each transmitter implements the ST protocol with the transmission time in each frame aligned to be within its assigned time slot; as a result, the maximum transmit-ratio, denoted by $1-\rho$, for each transmitter cannot exceed $1 / N$, which means that $\rho \geq$ $1-\frac{1}{N}$.

The protocol described above is illustrated in Fig. 3. Unlike the single-channel case where the transmitter can select any save-ratio $\rho$ in the interval $0 \leq \rho \leq 1$, in the case of TDMAST, $\rho$ is further constrained by $\rho \geq 1-\frac{1}{N}$ to ensure orthogonal transmissions by all transmitters. Due to this limitation, each transmitter may not be able to work at its individual minimum outage probability unless the corresponding optimal save-ratio $\rho^{*}$ satisfies $\rho^{*} \geq 1-\frac{1}{N}$ or $N \leq \frac{1}{1-\rho^{*}}$. In this case, ST protocol naturally extends to TDMA-ST with every transmitter operating at the optimal save-ratio $\rho^{*}$. However, if $N$ exceeds the threshold $\frac{1}{1-\rho^{*}}$, transmitters have to deviate from $\rho^{*}$ to maintain orthogonal transmissions. Next, we evaluate the performance of TDMA-ST for two types of source data at transmitters: Independent Data and Common Data.

\section{B. Independent Data}

First, consider the case where all transmitters send independent data packets to the FC in each frame, which are decoded separately at the FC. Under the symmetric setup, for a given $\rho$, all transmitters should have the same average outage performance. Consequently, the system-level outage performance in the case of independent data can be equivalently measured by that of the individual transmitter, i.e.,

$$
P_{\text {out }}^{s}=P_{\text {out }}\left(\rho, \eta, P_{c}\right) \text {. }
$$

We can further investigate the following two cases:

$$
\text { - } N \leq \frac{1}{1-\rho^{*}}
$$

In this case, the additional constraint due to TDMA, $\rho^{*} \geq$ $1-\frac{1}{N}$, is satisfied. Since $P_{\text {out }}^{s}$ is the same as that of the single-transmitter case, the system is optimized when all transmitters work at their individual minimum outage with save-ratio $\rho^{*}$. Thus, the minimum system outage probability is $P_{\text {out }}^{s *}=P_{\text {out }}^{*}\left(\eta, P_{c}\right)$.

- $N>\frac{1}{1-\rho^{*}}$

In this case, the TDMA constraint on $\rho^{*}$ is violated and thus we are not able to allocate all transmitters the saveratio $\rho^{*}$, which means that each transmitter has to deviate from its minimum outage point. Since in this case $\rho^{*}<$ $1-\frac{1}{N} \leq \rho$, the best strategy for each transmitter is to choose $\rho=1-\frac{1}{N}$. Thus, $P_{\text {out }}^{s *}=P_{\text {out }}\left(1-\frac{1}{N}, \eta, P_{c}\right)$.

To summarize, the optimal strategy for each transmitter in the case of independent data is given by

$$
\rho= \begin{cases}\rho^{*}, & N \leq \frac{1}{1-\rho^{*}} \\ 1-\frac{1}{N}, & N>\frac{1}{1-\rho^{*}}\end{cases}
$$

which implies that the number of transmitters should be kept below the reciprocal of the single-channel optimal transmitratio; otherwise, the system outage performance will degrade.

\section{Common Data}

Next, consider the case where all transmitters send identical data packets in each frame to the FC, which applies diversity combining techniques to decode the common data. For simplicity, we consider selection combining (SC) at the receiver, but similar results can be obtained for other diversity combining techniques [20]. With SC, the system outage probability is given by [20]

$$
P_{\text {out }}^{s}=\left(P_{\text {out }}\left(\rho, \eta, P_{c}\right)\right)^{N} .
$$

Similarly to the case of independent data, we can get exactly the same result for the optimal transmit strategy given in 22 for the common-data case, with which the minimum system outage probability is obtained as

$$
P_{\text {out }}^{s *}= \begin{cases}\left(P_{\text {out }}^{*}\left(\eta, P_{c}\right)\right)^{N}, & N \leq \frac{1}{1-\rho^{*}} \\ \left(P_{\text {out }}\left(1-\frac{1}{N}, \eta, P_{c}\right)\right)^{N}, & N>\frac{1}{1-\rho^{*}}\end{cases}
$$

From the above, it is evident that the system outage probability initially drops as $N$ increases, provided that $N \leq \frac{1}{1-\rho^{*}}$. However, when $N>\frac{1}{1-\rho^{*}}$, it is not immediately clear whether the system outage increases or decreases with $N$, since increasing $N$ improves the SC diversity, but also makes each transmitter deviate even further from its minimum outage save-ratio according to 22).

\section{NUMERICAL EXAMPLES}

In this section, we provide numerical examples to validate our claims. We assume that the energy harvesting rate $X$ follows a uniform distribution (unless specified otherwise) within $[0,100]$ (i.e., $P_{H}=100 \mathrm{~J} / \mathrm{s}$ ), and the channel is Rayleigh fading with exponentially distributed $\Gamma$ with parameter $\lambda=0.02$. We also assume the target transmission rate $R_{\text {req }}=\frac{Q}{T}=2$ bits $/ \mathrm{s}{ }^{7}$

Fig. 4 demonstrates how battery efficiency $\eta$ and circuit power $P_{c}$ affect the optimal save-ratio $\rho^{*}$ for the singlechannel case. As observed, larger $P_{c}$ and smaller $\eta$ result in larger $\rho^{*}$, i.e. shorter transmission time. Since the increment is more substantial along $P_{c}$ axis, circuit power has a larger influence on the optimal save-ratio compared with battery efficiency. $\rho^{*}(1,0)=0$ verifies the result of Lemma 3.1 for an ideal system, while $\rho^{*}(\eta, 0)$ along the line $P_{c}=0$ demonstrates the "phase transition" behavior stated in Lemma 3.2. The transition point is observed to be $\eta=0.541$, which can also be computed from (9).

Fig. 5] shows the optimal (minimum) outage probability $P_{\text {out }}^{*}\left(\eta, P_{c}\right)$ corresponding to $\rho^{*}$ in Fig. 4 Consistent with Proposition 3.1 $P_{\text {out }}^{*}\left(\eta, P_{c}\right)$ is observed to be monotonically decreasing with battery efficiency $\eta$ and monotonically increasing with circuit power $P_{c}$. Again, $P_{c}$ affects outage performance more significantly than $\eta$. From Fig. 5] we see

\footnotetext{
${ }^{7}$ This is normalized to a bandwidth of $1 \mathrm{~Hz}$, i.e. $R_{\text {req }}$ is the spectral efficiency in bis/s/Hz.
} 


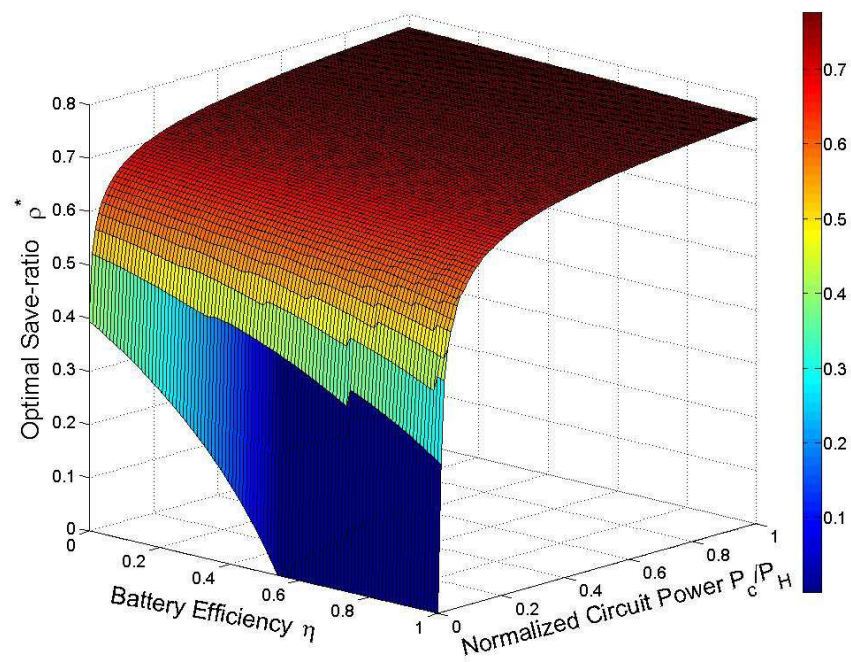

Fig. 4. Optimal save-ratio $\rho^{*}$

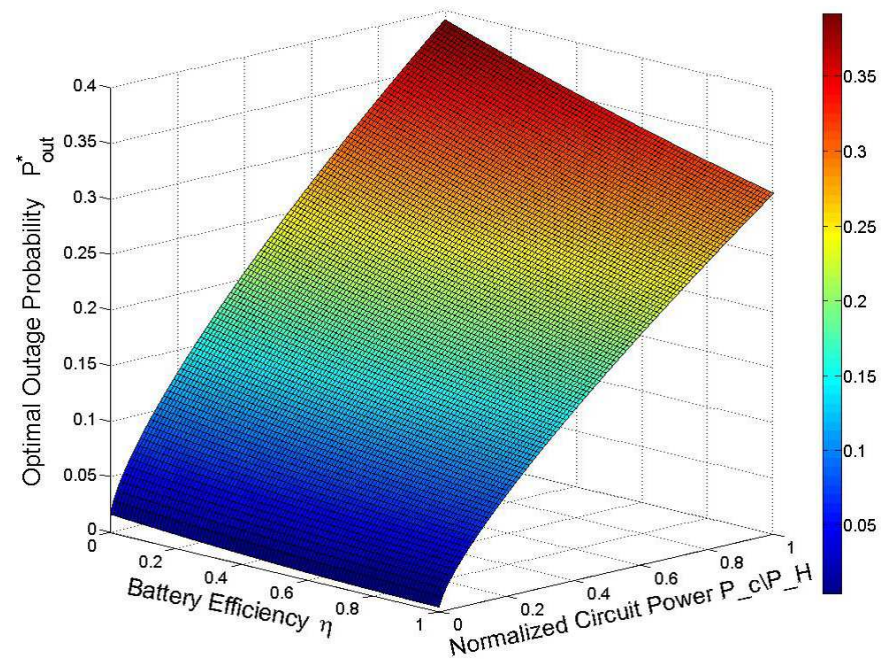

Fig. 5. Optimal outage probability $P_{\text {out }}^{*}$

that for a reasonable outage probability e.g. below $0.05, P_{c}$ has to be small and $\eta$ has to be close to 1 . Our results can thus be used to find the feasible region in the $\eta-P_{c}$ plane for a given allowable $P_{\text {out }}$.

Figs. 6 and 7 compare the outage performance with versus without save-ratio optimization. In Fig. 6 we fix the normalized circuit power $\frac{P_{c}}{P_{H}}=0.5$, while in Fig. 7 we fix the battery efficiency $\eta=0.8$. We observe that optimizing the save-ratio can significantly improve the outage performance. It is worth noting that $P_{\text {out }}$ has an approximately linear relationship with the normalized circuit power $\frac{P_{c}}{P_{H}}$ as observed in Fig. 7, which indicates that $P_{c}$ considerably affects the outage performance as stated previously.

In Fig. 8, the outage probability for an ideal system $(\eta=1$, $P_{c}=0$ ) is shown by numerically evaluating 177. By fixing the mean value of $P$ as $\mathbb{E}[P]=50 \mathrm{~J} / \mathrm{s}$ and varying $\beta$ for the Gamma distributed power from 1 to 5 , the resulting

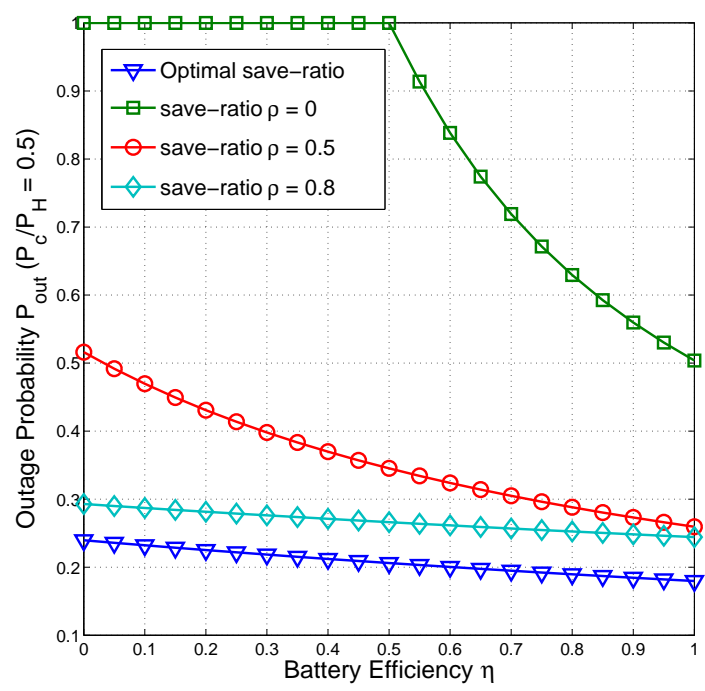

Fig. 6. Outage performance comparison: $\frac{P_{c}}{P_{H}}=0.5$

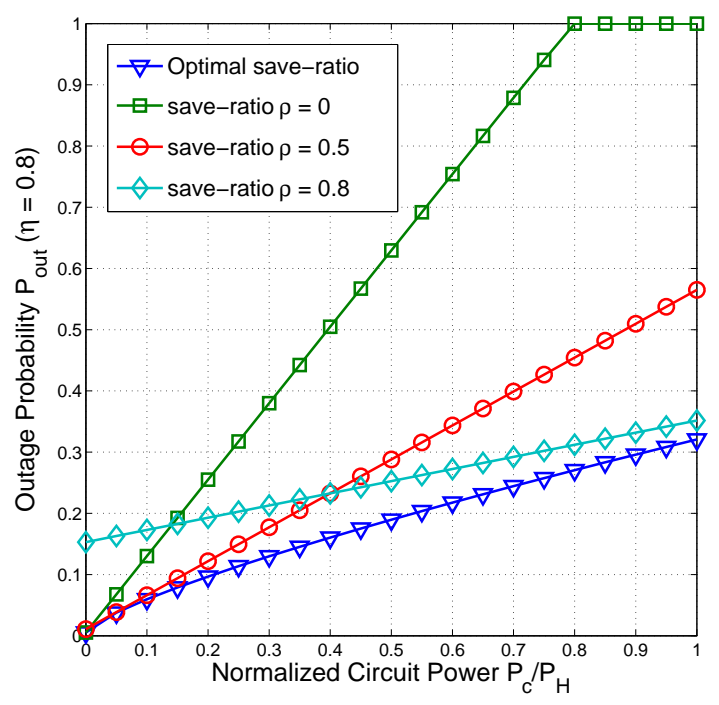

Fig. 7. Outage performance comparison: $\eta=0.8$

outage performance is compared with the case of constant power. As observed, the outage probability increases due to the existence of power randomness. As $\beta$ increases, the outage curve approaches the case of constant power. In Fig. 9 we also plot the outage probability for the ideal system with exponentially distributed power based on the approximation given in (19), as well as for a non-ideal system with the normalized circuit power $\frac{P_{c}}{\mathbb{E}[P]}=0.1$ and battery efficiency $\eta=0.8$. In comparison with the constant-power case, for the case of ideal system we observe that the high-SNR slope or diversity order with random power clearly converges much slower with SNR, which is in accordance with our analysis in Section IV] Furthermore, at $P_{\text {out }}=10^{-3}$, there is about 10 $\mathrm{dB}$ power penalty observed due to exponential random power, even with the same diversity order as the constant-power case. 


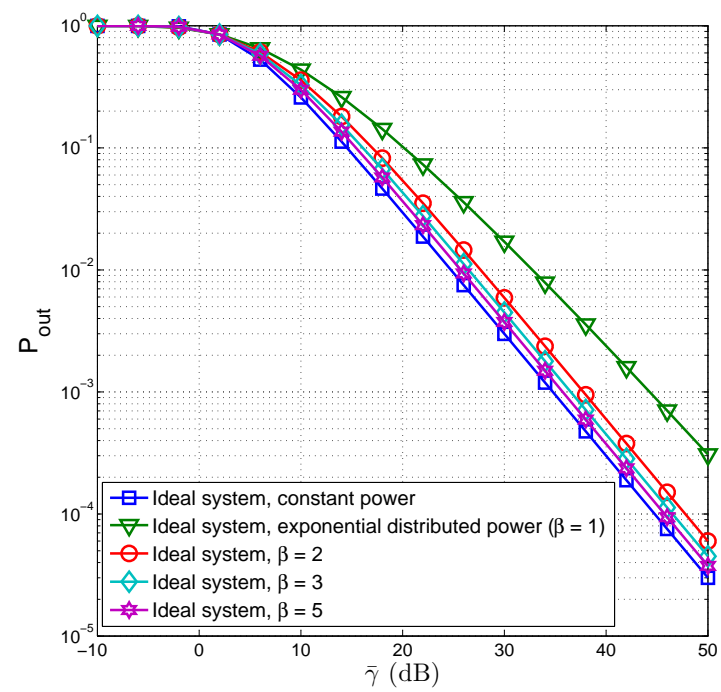

Fig. 8. Outage probability for an ideal $\left(\eta=1, P_{c}=0\right)$ system with constant power versus random power

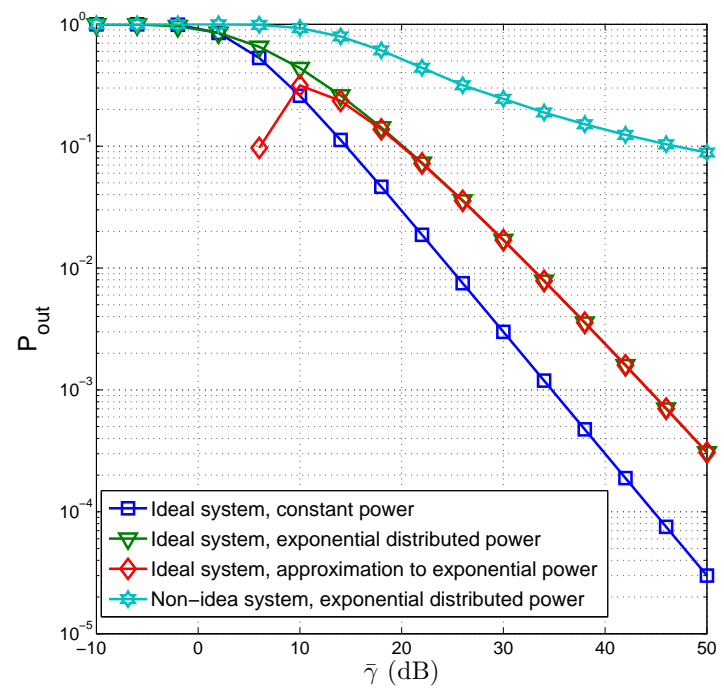

Fig. 9. Outage probability comparison for ideal $\left(\eta=1, P_{c}=0\right)$ versus non-ideal $\left(\eta=0.8, P_{c}=0.1 * \mathbb{E}[P]\right)$ systems

It is also observed that there is a small rising part for the outage approximation given in (19), since this approximation is only valid for sufficiently high SNR values $(\bar{\gamma}>10 \mathrm{~dB})$. Finally, it is worth noting that the outage probability for the non-ideal system eventually saturates with SNR (regardless of how small the noise power is or how large the SNR is), which indicates that the diversity order is zero for any non-ideal system.

Fig. 10 shows the outage performance for the case of multiple transmitters operating under the TDMA-ST protocol. We set the normalized circuit power $\frac{P_{c}}{P_{H}}=0.5$ and the battery efficiency $\eta=0.9$. Then, the optimal save-ratio $\rho^{*}$ for single-transmitter outage minimization can be obtained as 0.7930 by numerical search. Therefore, the threshold value for $N$ in the optimal rule of assigning save-ratio values in

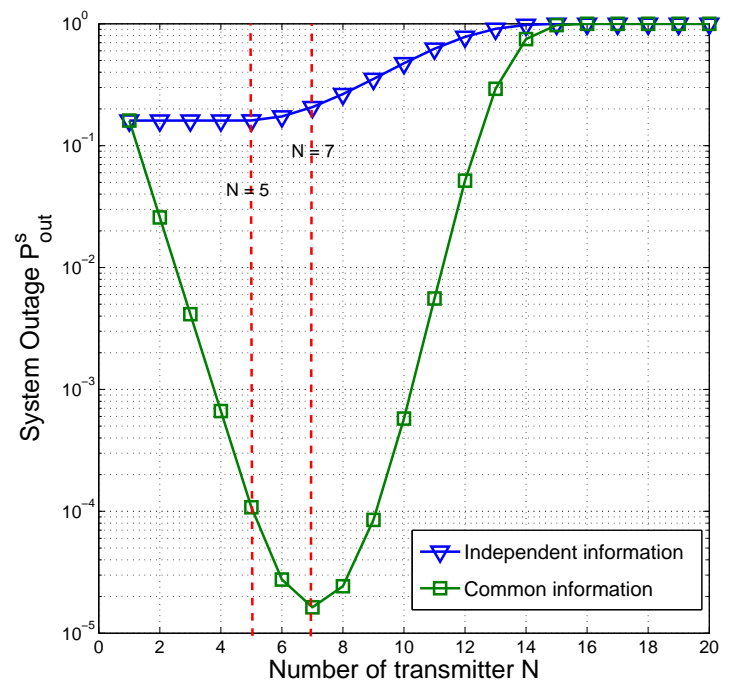

Fig. 10. Outage performance of multiple transmitters under TDMA-ST protocol, with $\frac{1}{1-\rho^{*}}=4.83$

(22) is $\frac{1}{1-\rho^{*}}=4.83$. For the case of independent data, it is observed that when $N \leq 4$, the system outage probability is constantly equal to the optimal single-transmitter outage probability $P_{\text {out }}^{*}\left(0.9,0.5 P_{H}\right)$; however, as $N>4$, the outage probability increases dramatically. In contrast, for the case of common data, it is observed that the system outage probability decreases initially as $N$ increases, even after the threshold value and until $N=7$, beyond which it starts increasing. This implies that there is an optimal decision on the number of transmitters to achieve the optimal outage performance.

\section{CONCLUSION}

In this paper, we studied a wireless system under practical energy harvesting conditions. Assuming a general model with non-ideal energy storage efficiency and transmit circuit power, we proposed a Save-then-Transmit (ST) protocol to optimize the system outage performance via finding the optimal save-ratio. We characterized how the optimal save-ratio and the minimum outage probability vary with practical system parameters. We compared the outage performance between random power and constant power under the assumption of Rayleigh fading channel. It is shown that random power considerably degrades the outage performance. Furthermore, we presented a TDMA-ST protocol for wireless networks with multiple transmitters. In particular, two types of source data are examined: independent data and common data. It is shown that if the number of transmitters is smaller than the reciprocal of the optimal transmit-ratio for single-transmitter outage minimization, each transmitter should work with its minimum outage save-ratio; however, when the number of transmitters exceeds this threshold, each transmitter has to deviate from its individual optimal operating point.

There are important problems that remain unaddressed in this paper and are worth investigating in the future. For example, we may consider the effect of different config- 
urations of battery/supercapacitor and MESD/SESD on the system performance. It is also interesting to investigate the information-theoretic limits for the ST protocol in the case of multiple transmitters using more sophisticated multiple-access techniques other than the simple TDMA.

\section{APPENDIX A}

PROOF OF PROPOSITION 3.1

According to the Fundamental Theorem of Calculus [26], we can derive the first derivative of $\hat{P}_{\text {out }}\left(\rho, \eta, P_{c}\right)$ in (7) with respect to $\eta, P_{c}$ and $\rho$ as

$$
\begin{aligned}
\frac{\partial \hat{P}_{\text {out }}}{\partial \beta} & =\left(\frac{P_{c}}{\frac{\rho}{1-\rho}+\eta}\right)^{\prime} f_{x}\left(\frac{P_{c}}{\frac{\rho}{1-\rho}+\eta}\right) \\
& -\left(\frac{P_{c}}{\frac{\rho}{1-\rho}+\eta}\right) f_{x}\left(\frac{P_{c}}{\frac{\rho}{1-\rho}+\eta}\right) F_{\Gamma}(\infty) \\
& +\int_{\frac{P_{c}}{\frac{\rho}{1-\rho}+\eta}}^{P_{H}} f_{X}(x) f_{\Gamma}[g(\cdot)] \frac{\partial g(\cdot)}{\partial \beta} d x \\
& =\int_{\frac{P_{H}}{\frac{\rho}{1-\rho}+\eta}}^{P_{H}} f_{X}(x) f_{\Gamma}[g(\cdot)] \frac{\partial g(\cdot)}{\partial \beta} d x
\end{aligned}
$$

where $\beta$ could be $\eta, P_{c}$ or $\rho$ and $g\left(\rho, \eta, P_{c}\right)=\frac{2^{\frac{Q}{(1-\rho) T}}-1}{x\left[\frac{\rho}{1-\rho}+\eta\right]-P_{c}}$.

It is easy to verify that $\frac{\partial g\left(\rho, \eta, P_{c}\right)}{\partial \eta}<0, \forall \eta \in[0,1]$ and $\frac{\partial g\left(\rho, \eta, P_{c}\right)}{\partial P_{c}}>0, \forall P_{c} \in[0, \infty]$. Therefore $\hat{P}_{\text {out }}\left(\rho, \eta, P_{c}\right)$ is strictly decreasing with battery efficiency $\eta$ and strictly increasing with circuit power $P_{c}$. Next, we are going to prove the monotonicity of $P_{\text {out }}$ and $P_{\text {out }}^{*}$ with battery efficiency $\eta$, where circuit power $P_{c}$ is treated as constant.

The condition $P_{H}>\frac{P_{c}}{\frac{\rho}{1-\rho}+\eta}$ in (6) could be expressed in terms of battery efficiency: $\eta>\frac{P_{c}}{P_{H}}-\frac{\rho}{1-\rho}$, then

$$
P_{\text {out }}=\left\{\begin{array}{ll}
1, & \eta \leq \frac{P_{c}}{P_{H}}-\frac{\rho}{1-\rho} \\
\hat{P}_{\text {out }}, & \eta>\frac{P_{c}}{P_{H}}-\frac{\rho}{1-\rho}
\end{array} .\right.
$$

Consider the following two cases:

- Suppose $\eta_{1}<\eta_{2}$ and $\frac{P_{c}}{P_{H}}-\frac{\rho}{1-\rho}>0$

- If $\frac{P_{c}}{P_{H}}-\frac{\rho}{1-\rho}<\eta_{1}<\eta_{2}$, then $P_{\text {out }}\left(\rho, \eta_{1}, P_{c}\right)=\hat{P}_{\text {out }}\left(\rho, \eta_{1}, P_{c}\right)$ and $P_{\text {out }}\left(\rho, \eta_{2}, P_{c}\right)=\hat{P}_{\text {out }}\left(\rho, \eta_{2}, P_{c}\right)$. Since $\hat{P}_{\text {out }}\left(\rho, \eta, P_{c}\right)$ is strictly decreasing with battery efficiency $\eta$, we have

$$
P_{\text {out }}\left(\rho, \eta_{1}, P_{c}\right)>P_{\text {out }}\left(\rho, \eta_{2}, P_{c}\right) .
$$

- If $\eta_{1} \leq \frac{P_{c}}{P_{H}}-\frac{\rho}{1-\rho}<\eta_{2}$, then $P_{\text {out }}\left(\rho, \eta_{1}, P_{c}\right)=1$ and $P_{\text {out }}\left(\rho, \eta_{2}, P_{c}\right)=\hat{P}_{\text {out }}\left(\rho, \eta_{2}, P_{c}\right)$. Therefore

$$
P_{\text {out }}\left(\rho, \eta_{1}, P_{c}\right)=1>P_{\text {out }}\left(\rho, \eta_{2}, P_{c}\right) .
$$

- If $\eta_{1}<\eta_{2} \leq \frac{P_{c}}{P_{H}}-\frac{\rho}{1-\rho}$, then $P_{\text {out }}\left(\rho, \eta_{1}, P_{c}\right)=$ $P_{\text {out }}\left(\rho, \eta_{2}, P_{c}\right)=1$, which means

$$
P_{\text {out }}\left(\rho, \eta_{1}, P_{c}\right)=P_{\text {out }}\left(\rho, \eta_{2}, P_{c}\right) .
$$

- Suppose $\eta_{1}<\eta_{2}$ and $\frac{P_{c}}{P_{H}}-\frac{\rho}{1-\rho} \leq 0$, we have $\frac{P_{c}}{P_{H}}-$ $\frac{\rho}{1-\rho} \leq \eta_{1}<\eta_{2}$. Then it could be easily verified that

$$
P_{\text {out }}\left(\rho, \eta_{1}, P_{c}\right)>P_{\text {out }}\left(\rho, \eta_{2}, P_{c}\right) \text {. }
$$

Combining all the above cases, we can conclude that $P_{\text {out }}\left(\rho, \eta, P_{c}\right)$ is a non-increasing function of battery efficiency $\eta$ given any non-zero circuit power $P_{c}$ for $\rho \in[0,1)$. Next, we proceed to prove the monotonicity of $P_{\text {out }}^{*}\left(\eta, P_{c}\right)$.

Assuming $\eta_{1}<\eta_{2}$ again, then we could argue that $\frac{P_{c}}{P_{H}}-\frac{\rho_{1}^{*}}{1-\rho_{1}^{*}}<\eta_{1}$ and $\frac{P_{c}}{P_{H}}-\frac{\rho_{2}^{*}}{1-\rho_{2}^{*}}<\eta_{2}$, where $\rho_{1}^{*}$ and $\rho_{2}^{*}$ are the optimal save-ratio for $\eta=\eta_{1}$ and $\eta=\eta_{2}$, respectively. Therefore we only need to consider two cases: $\max \left\{\frac{P_{c}}{P_{H}}-\frac{\rho_{1}^{*}}{1-\rho_{1}^{*}}, \frac{P_{c}}{P_{H}}-\frac{\rho_{2}^{*}}{1-\rho_{2}^{*}}\right\}<\eta_{1}<\eta_{2}$ and $\eta_{1} \leq$ $\max \left\{\frac{P_{c}}{P_{H}}-\frac{\rho_{1}^{*}}{1-\rho_{1}^{*}}, \frac{P_{c}}{P_{H}}-\frac{\rho_{2}^{*}}{1-\rho_{2}^{*}}\right\}<\eta_{2}$. From the arguments we have given for the proof of the monotonicity of $P_{\text {out }}$ we know that, under these two conditions we have

$$
P_{\text {out }}\left(\rho, \eta_{1}, P_{c}\right)>P_{\text {out }}\left(\rho, \eta_{2}, P_{c}\right) \text {. }
$$

Therefore,

$$
P_{\text {out }}^{*}\left(\eta_{1}, P_{c}\right)>P_{\text {out }}\left(\rho_{1}^{*}, \eta_{2}, P_{c}\right) \geq P_{\text {out }}^{*}\left(\eta_{2}, P_{c}\right)
$$

which completes the proof of the monotonicity for $P_{\text {out }}^{*}\left(\eta, P_{c}\right)$. With similar arguments, we could get the results regarding circuit power $P_{c}$. Proposition 3.1 is thus proved.

\section{APPENDIX B}

\section{PROOF OF LEMMA 3.1}

Since $F_{\Gamma}(\cdot)$ is non-negative and non-decreasing, we have

$$
a<b \Rightarrow F_{\Gamma}\left(\frac{a}{x}\right) \leq F_{\Gamma}\left(\frac{b}{x}\right)
$$

for any $x \in\left[0, P_{H}\right]$. Since $f_{X}(\cdot)$ is non-negative, this leads to

$$
\begin{aligned}
a<b & \Rightarrow \int_{0}^{P_{H}} f_{X}(x) F_{\Gamma}\left(\frac{a}{x}\right) d x \\
& \leq \int_{0}^{P_{H}} f_{X}(x) F_{\Gamma}\left(\frac{b}{x}\right) d x .
\end{aligned}
$$

Given the form of $P_{\text {out }}$ in Problem (P2), with $\rho$ appearing only in the numerator of the argument of $F_{\Gamma}(\cdot)$, we conclude that $P_{\text {out }}$ is a non-decreasing function of $g(\rho)=$ $\left(2^{\frac{Q}{(1-\rho) T}}-1\right)(1-\rho)$. Hence minimizing $g(\rho)$ is equivalent to minimizing $P_{\text {out }}$. The first and second derivatives of $g(\rho)$ are

$$
\begin{aligned}
g^{\prime}(\rho) & =2^{\frac{Q}{(1-\rho) T}}(\ln 2) \frac{Q}{(1-\rho) T}-2^{\frac{Q}{(1-\rho) T}}+1 \\
g^{\prime \prime}(\rho) & =2^{\frac{Q}{(1-\rho) T}}(\ln 2)^{2} \frac{Q^{2}}{T^{2}(1-\rho)^{3}}>0 \quad \text { since } Q>0 .
\end{aligned}
$$

Let $h(\rho)=g^{\prime}(\rho)$. From the second equation above, $h(\rho)$ is an increasing function. In the range $0 \leq \rho \leq 1, h(\rho)$ is thus minimized at $\rho=0$, i.e. the minimum of $g^{\prime}(\rho)$ is $h(0)$, given by

$$
\begin{aligned}
g_{\min }^{\prime} & =2^{\frac{Q}{T}}(\ln 2) \frac{Q}{T}-2^{\frac{Q}{T}}+1 \\
& =2^{\frac{Q}{T}}\left(\ln 2^{Q / T}-1\right)+1>0
\end{aligned}
$$


for $Q>0$. In other words, the smallest value that the gradient of $g(\rho)$ can take in the range $0 \leq \rho \leq 1$ for any feasible $Q$ is positive, which implies that $g(\rho)$ is increasing and therefore minimized at $\rho=0$, as claimed. The proof of Lemma 3.1 is thus completed.

\section{APPENDIX C}

PROOF OF LEMMA 3.2

To prove Property 1, we observe that as noted in the proof of Lemma 3.1. $P_{\text {out }}$ is a monotonic function of $g(\rho)=$ $\frac{\left(2 \frac{Q}{(1-\rho) T}-1\right)}{\left(\frac{\rho}{1-\rho}+\eta\right)}$ in Problem (P3), hence minimizing $g(\rho)$ leads to the same solution as minimizing $P_{\text {out }}$. The first derivative of $g(\rho)$ is

$$
\begin{aligned}
g^{\prime}(\rho) & =\frac{2^{\frac{Q}{(1-\rho) T}}(\ln 2) \frac{Q}{(1-\rho) T}[\rho+\eta(1-\rho)]-2^{\frac{Q}{(1-\rho) T}}+1}{[\rho+\eta(1-\rho)]^{2}} \\
& =\frac{u(\rho)}{[\rho+\eta(1-\rho)]^{2}} .
\end{aligned}
$$

It is clear in the above that the sign of $g^{\prime}(\rho)$ is the same as that of $u(\rho)$. Since $u(1)=+\infty$ and $u(\rho)$ is a differentiable function, if $u(0)$ is negative then there exists a value $\rho_{c} \in$ $(0,1)$ such that $u\left(\rho_{c}\right)=0=g^{\prime}\left(\rho_{c}\right)$. It is easily verified that $u^{\prime}(\rho)>0$; hence $\rho_{c}$ is the unique optimal value of $\rho$ in this case. Conversely, if there exists an $\rho_{c}$ such that $u\left(\rho_{c}\right)=0$, then $u(0)$ must be negative. Hence $u(0)<0$ is a necessary and sufficient condition for the optimal $\rho$ to lie in $(0,1)$.

The condition $u(0)<0$ translates into the following condition on $\eta$, which proves the first part of the lemma:

$$
\begin{aligned}
u(0)<0 & \Rightarrow 2^{\frac{Q}{T}}(\ln 2) \frac{Q}{T} \eta-2^{\frac{Q}{T}}+1<0 \\
& \Rightarrow \eta<\frac{2^{\frac{Q}{T}}-1}{2^{\frac{Q}{T}}(\ln 2) \frac{Q}{T}} .
\end{aligned}
$$

To prove the second point, suppose $\rho_{1}^{*}\left(\eta_{1}, 0\right)$ and $\rho_{2}^{*}\left(\eta_{2}, 0\right)$ are optimal save-ratios of (P3) for SESD efficiencies $\eta_{1}$ and $\eta_{2}$, where $\eta_{1}<\eta_{2}$. Then, $u\left(\rho_{1}^{*}, \eta_{1}\right)=0$ and $u\left(\rho_{2}^{*}, \eta_{2}\right)=0$. Since $\eta_{1}<\eta_{2}$ and $u(\rho, \eta)$ is an increasing function of $\eta$, we have $u\left(\rho_{1}^{*}, \eta_{2}\right)>0$. Combining what we have that $u(\rho, \eta)$ is an increasing function of $\rho, u\left(\rho_{2}^{*}, \eta_{2}\right)=0$ and $u\left(\rho_{1}^{*}, \eta_{2}\right)>0$, we may conclude $\rho_{2}^{*}\left(\eta_{2}, 0\right)<\rho_{1}^{*}\left(\eta_{1}, 0\right)$. Lemma 3.2 is thus proved.

\section{APPENDIX D}

PROOF OF LEMMA 3.3

According to the proof of Proposition 3.1 the first derivative of $\hat{P}_{\text {out }}\left(\rho, \eta, P_{c}\right)$ with respect to $\eta, P_{c}$ and $\rho$ is,

$$
\frac{\partial \hat{P}_{\text {out }}}{\partial \beta}=\int_{\frac{P_{c}}{\frac{\rho}{1-\rho}+\eta}}^{P_{H}} f_{X}(x) f_{\Gamma}[g(\cdot)] \frac{\partial g(\cdot)}{\partial \beta} d x
$$

where $\beta$ could be $\eta, P_{c}$ or $\rho$ and $g\left(\rho, \eta, P_{c}\right)=\frac{2^{\frac{Q}{(1-\rho) T}}-1}{x\left[\frac{\rho}{1-\rho}+\eta\right]-P_{c}}$. Furthermore, we have

$$
\begin{aligned}
\frac{\partial g(\rho)}{\partial \rho} & =\frac{2^{\frac{Q}{(1-\rho) T}}(\ln 2) \frac{Q}{(1-\rho) T}\left[\rho+\eta(1-\rho)-(1-\rho) \frac{P_{c}}{x}\right]}{x\left[\rho+\eta(1-\rho)-(1-\rho) \frac{P_{c}}{x}\right]^{2}} \\
& -\frac{2^{\frac{Q}{(1-\rho) T}}-1}{x\left[\rho+\eta(1-\rho)-(1-\rho) \frac{P_{c}}{x}\right]^{2}} \\
& =\frac{v(\rho)}{x\left[\rho+\eta(1-\rho)-(1-\rho) \frac{P_{c}}{x}\right]^{2}} .
\end{aligned}
$$

With similar arguments about $u(\rho)$ in the proof of Lemma 3.2 we claim that $v(0)<0, \forall x \in\left(\frac{P_{c}}{\frac{\rho}{1-\rho}+\eta}, P_{H}\right]$ is a sufficient condition of having $\rho^{*}\left(\eta, P_{c}\right)>0$ while $P_{c}<\eta P_{H}$.

Since $v(0)$ is an increasing function of $x$, the condition $v(0)<0, \forall x \in\left(\frac{P_{c}}{\frac{\rho}{1}+\eta}, P_{H}\right]$ translates into the following condition on $\eta$ and ${ }^{\frac{\rho}{P}}{ }_{c}^{\rho}$

$$
\begin{aligned}
v(0) & =2^{\frac{Q}{T}}(\ln 2) \frac{Q}{T}\left(\eta-\frac{P_{c}}{x}\right)-2^{\frac{Q}{T}}+1 \\
& <2^{\frac{Q}{T}}(\ln 2) \frac{Q}{T}\left(\eta-\frac{P_{c}}{P_{H}}\right)-2^{\frac{Q}{T}}+1<0 \\
& \Longrightarrow 0<\eta-\frac{P_{c}}{P_{H}}<\frac{2^{\frac{Q}{T}}-1}{2^{\frac{Q}{T}}(\ln 2) \frac{Q}{T}} .
\end{aligned}
$$

Combined with the fact that $\rho^{*}\left(\eta, P_{c}\right)>\frac{\frac{P_{c}}{P_{H}}-\eta}{1-\eta+\frac{P_{c}}{P_{H}}}$ when $P_{c} \geq$ $\eta P_{H}$, we may conclude

$$
\rho^{*}\left(\eta, P_{c}\right)>0, \quad \eta-\frac{P_{c}}{P_{H}}<\frac{2^{\frac{Q}{T}}-1}{2^{\frac{Q}{T}}(\ln 2) \frac{Q}{T}} .
$$

Lemma 3.3 is thus proved.

\section{APPENDIX E}

\section{PROOF OF LEMMA 4.1}

Let $Z=P \Gamma$, where $P$ and $\Gamma$ are exponential random variables with mean $\lambda_{p}$ and $\lambda_{\gamma}$ respectively. Then the PDF of $Z$ could be derived as follows,

$$
\begin{aligned}
F_{Z}(z) & =\operatorname{Pr}\{P \Gamma \leq z\} \\
& =1-\frac{1}{\lambda_{p}} \int_{0}^{\infty} e^{-\frac{z}{p \lambda \gamma}} e^{-\frac{p}{\lambda_{p}}} d p \\
& =1-2 \sqrt{\frac{z}{\lambda_{p} \lambda_{\gamma}}} K_{1}\left(2 \sqrt{\frac{z}{\lambda_{p} \lambda_{\gamma}}}\right)
\end{aligned}
$$

where $K_{1}(x)$ is the first-order modified Bessel function of the second kind and the last equality is given by [25, §3.324.1]:

$$
\int_{0}^{\infty} \exp \left(-\frac{\beta}{4 x}-\gamma x\right) d x=\sqrt{\frac{\beta}{\gamma}} K_{1}(\sqrt{\beta \gamma})
$$


where $\Re(\beta) \geq 0, \Re(\gamma) \geq 0$. Let $M=\frac{1}{\sqrt{\lambda_{p} \lambda_{\gamma}}}$. Taking the derivative of $F(z)$ yields

$$
\begin{aligned}
f(z) & =M\left\{-\frac{1}{\sqrt{z}} K_{1}(2 M \sqrt{z})-2 \sqrt{z}\left(K_{1}(2 M \sqrt{z})\right)^{\prime}\right\} \\
& =M\left\{-\frac{1}{\sqrt{z}} K_{1}(2 M \sqrt{z})\right. \\
& \left.-2 \sqrt{z}\left(-K_{0}(2 M \sqrt{z})-\frac{1}{2 M \sqrt{z}} K_{1}(2 M \sqrt{z})\right) \frac{M}{\sqrt{z}}\right\} \\
& =2 M^{2} K_{0}(2 M \sqrt{z}) \\
& =\frac{2}{\lambda_{p} \lambda_{\gamma}} K_{0}\left(2 \sqrt{\frac{z}{\lambda_{p} \lambda_{\gamma}}}\right)
\end{aligned}
$$

where $\frac{\partial K_{v}(z)}{\partial z}=-K_{v-1}(z)-\frac{v}{z} K_{v}(z)$.

Next, we characterize the outage probability using (33). According to (15), we have

$$
\begin{aligned}
P_{\text {out }}^{*} & =\operatorname{Pr}[P \Gamma<C] \\
& =\int_{0}^{C} \frac{2}{\lambda_{p} \lambda_{\gamma}} K_{0}\left(2 \sqrt{\frac{z}{\lambda_{p} \lambda_{\gamma}}}\right) d z .
\end{aligned}
$$

Let $X=\frac{z}{\lambda_{p} \lambda_{\gamma}}$ and $D=\frac{C}{\lambda_{p} \lambda_{\gamma}}$. We then have

$$
P_{\text {out }}^{*}=2 \int_{0}^{D} K_{0}(2 \sqrt{x}) d x .
$$

Using the series presentation [25, $\S 8.447 .3$ ], we have

$$
K_{0}(x)=-\ln \left(\frac{x}{2}\right) I_{0}(x)+\sum_{k=0}^{\infty} \frac{x^{2 k}}{2^{2 k}(k !)^{2}} \psi(k+1)
$$

with the series expansion for the modified Bessel function given by

$$
I_{0}(x)=\sum_{k=0}^{\infty} \frac{x^{2 k}}{2^{2 k}(k !)^{2}} .
$$

(35) could be expanded as

$P_{\text {out }}^{*}=\sum_{k=0}^{\infty} \frac{2}{(k !)^{2}}\left[-\frac{1}{2} \int_{0}^{D} x^{k} \ln x d x+\psi(k+1) \int_{0}^{D} x^{k} d x\right]$

where

$$
\psi(x)=\frac{d}{d x} \ln \Gamma(x)=\frac{\Gamma(x)^{\prime}}{\Gamma(x)}
$$

is the digamma function [24]. Since the two integrals in 38] could be evaluated as

$$
\begin{aligned}
\int_{0}^{D} x^{k} d x & =\frac{D^{k+1}}{k+1} \\
\int_{0}^{D} x^{k} \ln x d x & =\left.x^{k+1}\left(\frac{\ln x}{k+1}-\frac{1}{(k+1)^{2}}\right)\right|_{x=0} ^{x=D} \\
& =D^{k+1}\left(\frac{\ln D}{k+1}-\frac{1}{(k+1)^{2}}\right)
\end{aligned}
$$

where $\lim _{x \rightarrow 0}(x \ln x)=0$. Then we have

$$
P_{\text {out }}^{*}=\sum_{k=0}^{\infty} \frac{2}{(k !)^{2}} \frac{D^{k+1}}{k+1}\left[-\frac{1}{2}\left(\ln D-\frac{1}{k+1}\right)+\psi(k+1)\right] \text {. }
$$

Since $D=\frac{C}{\lambda_{p} \lambda_{\gamma}}=\frac{C \sigma_{n}^{2}}{\lambda_{p} \sigma_{h}^{2}}=\frac{C}{\bar{\gamma}}$, (18) follows. Lemma 4.1 is thus proved.

\section{REFERENCES}

[1] A. Sinha and A. Chandrakasan, "Dynamic power management in wireless sensor networks," IEEE Design Test Comp., vol. 18, no. 2, pp. 62-74, Mar./Apr. 2001.

[2] I. F. Akyidiz, W. Su, Y. S. Subramaniam, and E. Cayirei, "A survey on sensor networks," IEEE Commun. Mag., vol. 40, pp. 102-114, Aug. 2002.

[3] J. Yang and S. Ulukus, "Optimal packet scheduling in an energy harvesting communication system," IEEE Trans. Commun., vol. 60, no. 1, pp. 220-230, Jan. 2012.

[4] K. Tutuncuoglu and A. Yener, "Optimum transmission policies for battery limited energy harvesting nodes," IEEE Trans. Wireless Commun., vol. 11, no. 3, pp. 1180-1189, Mar. 2012.

[5] B. Devillers and D. Gunduz, "A general framework for the optimization of energy harvesting communication systems with battery imperfections," J. Commun. and Netw., Special Issue on Energy Harvesting in Wireless Networks, vol. 14, no. 2, pp. 130-139, Apr. 2012.

[6] K. Tutuncuoglu and A. Yener, "Optimal power policy for energy harvesting transmitters with inefficient energy storage," in Proc. Annual Conference on Information Sciences and Systems (CISS), Mar. 2012.

[7] C. Huang, R. Zhang, and S. Cui, "Throughput maximization for the gaussian relay channel with energy harvesting constraints," to appear in IEEE J. Sel. Areas Commun., special issue on theories and methods for advanced wireless relays. Available at [arXiv:1109.0724].

[8] O. Ozel, K. Tutuncuoglu, J. Yang, S. Ulukus, and A. Yener, "Transmission with energy harvesting nodes in fading wireless channels: optimal policies," IEEE J. Sel. Areas Commun., vol. 29, no. 8, pp. 1732-1743, Sep. 2011.

[9] C. K. Ho and R. Zhang, "Optimal energy allocation for wireless communications with energy harvesting constraints," IEEE Trans. Signal Process., vol. 60, no. 9, pp. 4808-4818, Sep. 2012.

[10] A. C. Fu, E. Modiano, and J. N. Tsitsiklis, "Optimal energy allocation and admission control for communications satellites," IEEE/ACM Trans. Netw., vol. 11, no. 3, Jun. 2003.

[11] V. Sharma, U. Mukherji, V. Joseph, and S. Gupta, "Optimal energy management policies for energy harvesting sensor nodes," IEEE Trans. Wireless Commun., vol. 9, pp. 1326-1336, Apr. 2010.

[12] L. Ren-Shiou, P. Sinha., and C. E. Koksal, "Joint energy management and resource allocation in rechargeable sensor networks," in Proc. IEEE INFOCOM, San Diego, CA, pp. 1-9, Mar. 2010.

[13] F. Iannello, O. Simeone, and U. Spagnolini, "Medium access control protocols for wireless sensor networks with energy harvesting," IEEE Trans. Commun., vol. 60, no. 5, pp. 1381-1389, May. 2012.

[14] V. Sharma, U. Mukherji, and V. Joseph, "Efficient energy management policies for networks with energy harvesting sensor nodes," in Proc. Annual Allerton Conference on Communication, Control and Computing, USA, Sep. 2008.

[15] O. Ozel and S. Ulukus, "Information-theoretic analysis of an energy harvesting communication system," in Proc. IEEE PIMRC, Sep. 2010.

[16] R. Rajesh, V. Sharma, and P. Viswanath, "Capacity of fading Gaussian channel with an energy harvesting sensor node," in Proc. IEEE Global Commun. Conf. (Globecom), Dec. 2011.

[17] M. Jayalakshmi and K. Balasubramanian, "Simple capacitors to supercapacitors - an overview," Int. J. Electrochem. Sci., vol. 3, pp. 1196-1217, 2008.

[18] S. Sudevalayam and P. Kulkarni, "Energy harvesting sensor nodes: survey and implications," IEEE Communications Surveys \& Tutorials, vol. 13, pp. 443-461, 2011

[19] G. Miao, N. Himayat, and G. Y. Li, "Energy-efficient link adaptation in frequency-selective channels," IEEE Trans. Commun., vol. 58, no. 20, Feb. 2010.

[20] D. Tse and P. Viswanath, Fundamentals of Wireless Communications. Cambridge, UK: Cambridge University Press, 2005.

[21] M. Springer, The Algebra of Random Variables. Wiley New York, 1979.

[22] S. A. Halim and H. Yanikomeroglu, "On the approximation of the generalized-k distribution by a gamma distribution for modeling composite fading channels," IEEE Trans. Wireless Commun., vol. 7, no. 7, Jul. 2008 .

[23] S. Nadarajah, "Exact distribution of the product of $\mathrm{m}$ gamma and n Pareto random variables," Journal of Computational and Applied Mathematics, vol. 235, no. 15, pp. 4496-4512, June 2011. 
[24] M. Abramowitz and I. A. Stegun, Handbook of Mathematical Functions with Formulas, Graphs, and Mathematical Tables. Courier Dover Publications, 1965.

[25] I. Gradshteyn and I. Ryzhik, Table of integrals, series, and products. 4th ed. Academic Press, Inc., 1980.

[26] R. Walter, Real and Complex Analysis. 3rd ed. New York: McGraw-Hill Book, 1987. 